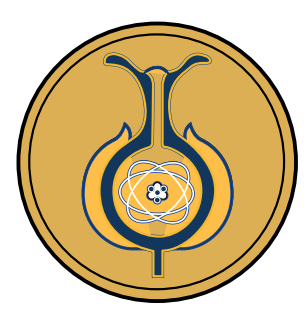

\title{
Efecto de un difusor tipo wind lens en flujo turbulento
}

\author{
Effect of a Wind Lens diffuser on turbulent flow \\ Efeito de um difusor tipo wind lens em fluxo turbulento
}

Gustavo Richmond-Navarro ${ }^{1}$, Pedro Casanova-Treto ${ }^{2}$, Franklin Hernández-Castro ${ }^{3,4}$

Received: Oct/8/2020 • Accepted: Feb/15/2021 • Published: Jul/31/2021

\section{Resumen}

El objetivo fue medir el efecto de un concentrador de flujo tipo wind lens en el caso de flujo turbulento. El estudio se realizó en el túnel de viento de capa límite del Instituto de Investigación en Mecánica Aplicada de la Universidad de Kyushu, Japón, entre marzo y mayo de 2019. Se utilizó un anemómetro de hilo caliente con un mecanismo móvil para realizar tres pruebas. Primero se mide la velocidad en el eje del túnel, sin la rejilla de turbulencia instalada. En segundo lugar, se coloca la rejilla y se mide la intensidad de turbulencia en el eje del túnel de viento. En tercer lugar, se determina el efecto del WL en la velocidad del viento incidente, a diferentes niveles de intensidad de turbulencia. La velocidad del viento en el túnel sin la rejilla de turbulencia es casi constante, alrededor de $9.6 \mathrm{~m} / \mathrm{s}$. Al colocar la rejilla se registró en el eje del túnel de viento una intensidad de turbulencia decreciente, desde un valor de $28.6 \%$ a $500 \mathrm{~mm}$ de la rejilla, hasta un $5 \%$ de intensidad de turbulencia a $3100 \mathrm{~mm}$ de la rejilla. Cuando se midió el efecto del wind lens en flujo turbulento, se obtuvo un aumento del $20 \%$ o más en la velocidad del viento. El wind lens demostró ser adecuado para turbinas eólicas que operan en flujo turbulento, acrecentó la velocidad del viento en todas las pruebas, generando un mayor aumento a mayor intensidad de turbulencia.

Palabras clave: Wind lens; concentrador de flujo; flujo turbulento; intensidad de turbulencia.

\section{Abstract}

This article is aimed to measure the effect of a wind lens diffuser on turbulent flow. The study was conducted in the boundary layer wind tunnel of the Research Institute for Applied Mechanics at Kyushu University, Japan, between March and May 2019. A hot wire anemometer with a moving mechanism was used to conduct three tests. First, a characterization of the flow inside the wind tunnel was measured without the grid. Second, the turbulence intensity in the wind tunnel axis was measured this time with the grid in place. Third, the wind lens effect on the incident wind speed was determined at different levels of turbulence intensity. The wind speed in the tunnel without the turbulence grid was almost constant, approximately $9.6 \mathrm{~m} / \mathrm{s}$. When the grid was placed, a decreasing turbulence intensity was recorded in the axis of the wind

\footnotetext{
Gustavo Richmond-Navarro, $\square$ grichmond@tec.ac.cr, (D) https://orcid.org/0000-0001-5147-5952

Pedro Casanova-Treto, $\triangle$ pedro.casanova@ucr.ac.cr, (iD https://orcid.org/0000-0001-8508-6293

Franklin Hernández-Castro, $\$ franhernandez@tec.ac.cr, (1) https://orcid.org/0000-0003-3589-4588

1 Laboratorio de Investigación en Energía Eólica, Escuela de Ingeniería Electromecánica, Instituto Tecnológico de Costa Rica, Cartago, Costa Rica.

2 Instituto de Investigaciones en Ingeniería (INII), Facultad de Ingeniería, Universidad de Costa Rica, San José, Costa Rica.

3 iReal research group, Escuela de Ingeniería en Diseño Industrial, Instituto Tecnológico de Costa Rica, Cartago, Costa Rica.

4 Hochschule für Gestaltung Schwäbisch Gmünd, Schwabisch Gmund, Germany
} 
tunnel, from $28.6 \%$ at $500 \mathrm{~mm}$ from the grid to $5 \%$ turbulence intensity at $3100 \mathrm{~mm}$ from the grid. When the effect of the wind lens was measured on the turbulent flow, wind speed increased up to $20 \%$. The wind lens proved suitable for wind turbines operating in turbulent flow, by increasing wind speed in all tests and generating a greater increase in the conditions with greater turbulence intensity.

Keywords: Wind lens; diffuser; turbulent flow; turbulence intensity

\section{Resumo}

0 objetivo foi medir 0 efeito de um concentrador de fluxo tipo wind lens no caso de fluxo turbulento. 0 estudo foi realizado no túnel de vento de camada limite do Instituto de Pesquisa em Mecânica Aplicada da Universidade de Kyushu, Japão, entre março e maio de 2019. Foi utilizado um anemômetro de fio quente com um mecanismo móvel para a realização de três testes. Primeiro, mede-se a velocidade no eixo do túnel, sem a grelha de turbulência instalada. Segundo, coloca-se a grelha e mede-se a intensidade de turbulência no eixo do túnel de vento. Em terceiro lugar, determina-se o efeito do WL na velocidade do vento incidente, em diferentes níveis de intensidade de turbulência. A velocidade do vento no túnel sem a grelha de turbulência é quase constante, aproximadamente $9,6 \mathrm{~m} / \mathrm{s}$. Ao colocar a grelha foi registrada no eixo do túnel de vento uma intensidade de turbulência decrescente, desde um valor de $28,6 \%$ a $500 \mathrm{~mm}$ da grelha, até $5 \%$ de intensidade de turbulência a $3100 \mathrm{~mm}$ da grelha. Ao medir 0 efeito do wind lens em fluxo turbulento, obteve-se um aumento de $20 \%$ ou mais na velocidade do vento. 0 wind lens mostrou ser ideal para turbinas eólicas que operam em fluxo turbulento, aumentou a velocidade do vento em todas os testes, gerando um maior aumento em uma maior intensidade de turbulência.

Palavras-chave: Wind lens; concentrador de fluxo; fluxo turbulento; intensidade de turbulência.

\section{Introducción}

El aprovechamiento de la energía del viento mediante turbinas eólicas presenta hoy en día una amplia variedad de opciones como lo son las turbinas tradicionales de eje vertical y horizontal, así como otras alternativas entre las que se pueden mencionar los postes oscilantes (El-Shahat, Hasan, \& Wu, 2018); turbinas tipo tornillo (Jang, Kim, Hwang, Paek, Kim, \& Baek, 2019); turbinas con una única superficie (Aouini, 2012); turbinas de eje horizontal que utilizan cilindros en vez de aspas (RichmondNavarro, Calderon-Munoz, LeBoeuf, \& Castillo, 2017); turbinas que aprovechan la convección debida a diferencias de temperatura (Watanabe, Fukutomi, Ohya, \& Uchida, 2020), y turbinas con concentradores de flujo, las cuales alcanzan altos coeficientes de potencia (Keramat Siavash, Najafi, Tavakkoli Hashjin, Ghobadian, \& Mahmoodi, 2020), pueden operar flotando en el agua (Zhu, Sueyoshi, Hu, \& Yoshida, 2019), ofrecen beneficios de incremento de potencia en sistemas multirotor (Yuji, \& Koichi, 2019) y aumentan la eficiencia aun en condiciones de baja velocidad de viento (Riyanto et al., 2019).

Entre las diferentes opciones de concentradores de flujo para turbinas eólicas, existe un modelo particular llamado wind lens (WL), que ofrece beneficios como incremento en la potencia de salida (Clements, \& Chowdhury, 2019; Heikal, et al., 2018), control de la orientación de la turbina por el anillo del mismo WL (Ohya, \& Karasudani, 2010), reducción de ruido 
(Ohya, Karasudani, Nagai, \& Watanabe, 2017; Takahashi, Hata, Ohya, Karasudani, \& Uchida, 2012) y mejora en la seguridad (Khamlaj, \& Rumpfkeil, 2018). Además, el WL es más amigable con las aves que las turbinas convencionales como se aprecia en $\mathrm{Hu}, \&$ Wang (2015); lo que lo hace atractivo para su uso en zonas boscosas que, de acuerdo con SAGE Journals (2006), poseen bajo potencial eólico y alta turbulencia.

Precisamente en Clements, \& Chowdhury (2019) se evalúa el desempeño del WL en ambiente de alta turbulencia y velocidades relativamente bajas, y se concluye que existe una mejora cuantitativa de la turbina con WL frente a la turbina convencional, en flujo turbulento. Un resultado similar se reporta en Kosasih, \& Saleh Hudin (2016), donde se evalúa la influencia del flujo turbulento en una micro turbina eólica equipada con un difusor, tanto de forma experimental en túnel de viento como mediante dinámica de fluidos computacional (CFD). Otros estudios señalan desventajas del WL como aumento de los esfuerzos en las aspas (Wang, Matsubara, Hu, Odahara, Nagai, Karasutani, \& Ohya, 2015) y, en contraste con investigaciones previamente citadas, discuten un aumento del ruido (Hashem, Mohamed, \& Hafiz, 2017).

Con el fin de caracterizar el efecto del WL, existen en estudios del flujo a través del propio WL sin presencia de la turbina, donde se aprecia cómo se concentran las líneas de flujo (Heikal, et al., 2018; Khamlaj, \& Rumpfkeil, 2018; Maftouni, \& Parsa, 2019; Nasution, \& Purwanto, 2011; Ohya, \& Karasudani, 2010); sin embargo, no ha sido reportado el caso de mediciones de la velocidad del viento en un campo de flujo turbulento con el WL y sin este, para evaluar el efecto de este concentrador en condiciones de turbulencia.
Por lo anterior, el objetivo de esta investigación es describir la velocidad del viento en un flujo turbulento, en diferentes secciones transversales de un túnel de viento, con presencia y ausencia de un concentrador de flujo del tipo WL.

\section{Metodología}

Se realiza una investigación del tipo experimental, con un enfoque cuantitativo. Los experimentos se llevaron a cabo entre el 01 de marzo y el 31 de mayo de 2019, en el túnel de viento de capa límite del Instituto de Investigación en Mecánica Aplicada (RIAM, por sus siglas en inglés Research Institute for Applied Mechanics) de la Universidad de Kyushu, Japón.

Además de la velocidad del viento, una variable principal de esta investigación es la intensidad de turbulencia (IT), que se calcula como la desviación estándar de la muestra entre el promedio de las mediciones de velocidad de viento tomadas en un mismo periodo (Burton, Jenkins, Sharpe, \& Bossanyi, 2011). Luego, el resultado se multiplica por 100 para expresar la IT como un porcentaje, como se muestra en la ecuación 1.

$$
\mathrm{IT}=\frac{\sqrt{\frac{\sum_{i=1}^{n}\left(v_{i}-\bar{v}\right)^{2}}{n-1}}}{\frac{1}{n} \sum_{i=1}^{n} v_{i}} \times 100
$$

Donde $v_{i}$ representa cada medición, $\bar{v}$ es el promedio de las mediciones y $n$ es la cantidad total de datos recolectados, que para cada punto de medición corresponde al producto de la frecuencia de muestreo por el tiempo de muestreo.

A continuación, se detalla el equipamiento y las pruebas realizadas en esta investigación. 


\section{Equipamiento}

El túnel de viento empleado se muestra esquemáticamente en la Figura 1. La sección de pruebas del túnel posee $15 \mathrm{~m}$ de largo, 3.6 $\mathrm{m}$ de ancho y $2 \mathrm{~m}$ de alto. Los detalles técnicos del túnel se pueden consultar en Ohya (2019). En la sección de pruebas hay 9 paneles removidos con el objetivo de minimizar el efecto de bloqueo (Göltenbott, Ohya, Yoshida, \& Jamieson, 2017; Ohya, 2019), los paneles laterales y el panel superior de los tramos 3, 4 y 5 de la Figura 1. En el caso del panel 3 del lado derecho (visto en la dirección del flujo), está removido solo parcialmente.

La turbulencia se generó con una rejilla de madera, la cual se muestra instalada, junto con el resto del montaje experimental, dentro del túnel de viento, en la Figura 2. En el recuadro inferior derecho de la misma Figura 2 se detallan las dimensiones específicas de la rejilla, que son $\mathrm{M}=156.2 \mathrm{~mm}, \mathrm{~b}=43.5 \mathrm{~mm} \mathrm{y} \mathrm{t}=29.9 \mathrm{~mm}$.

En la Figura 3 se resaltan algunos detalles de la Figura 2, como lo es el WL, la ubicación del anemómetro de hilo caliente, que está montado en un mecanismo móvil dentro del WL y el anemómetro ultrasónico en el piso del túnel del lado derecho, el cual se empleó para la calibración del anemómetro de hilo caliente. Dicha calibración consiste en determinar la función lineal que correlaciona el voltaje que registra el anemómetro de hilo caliente, con el valor de velocidad del viento registrada por el anemómetro ultrasónico. Para esta calibración se acciona el propio túnel de viento y se regula la velocidad de rotación de los ventiladores hasta que en el anemómetro ultrasónico se mida una velocidad

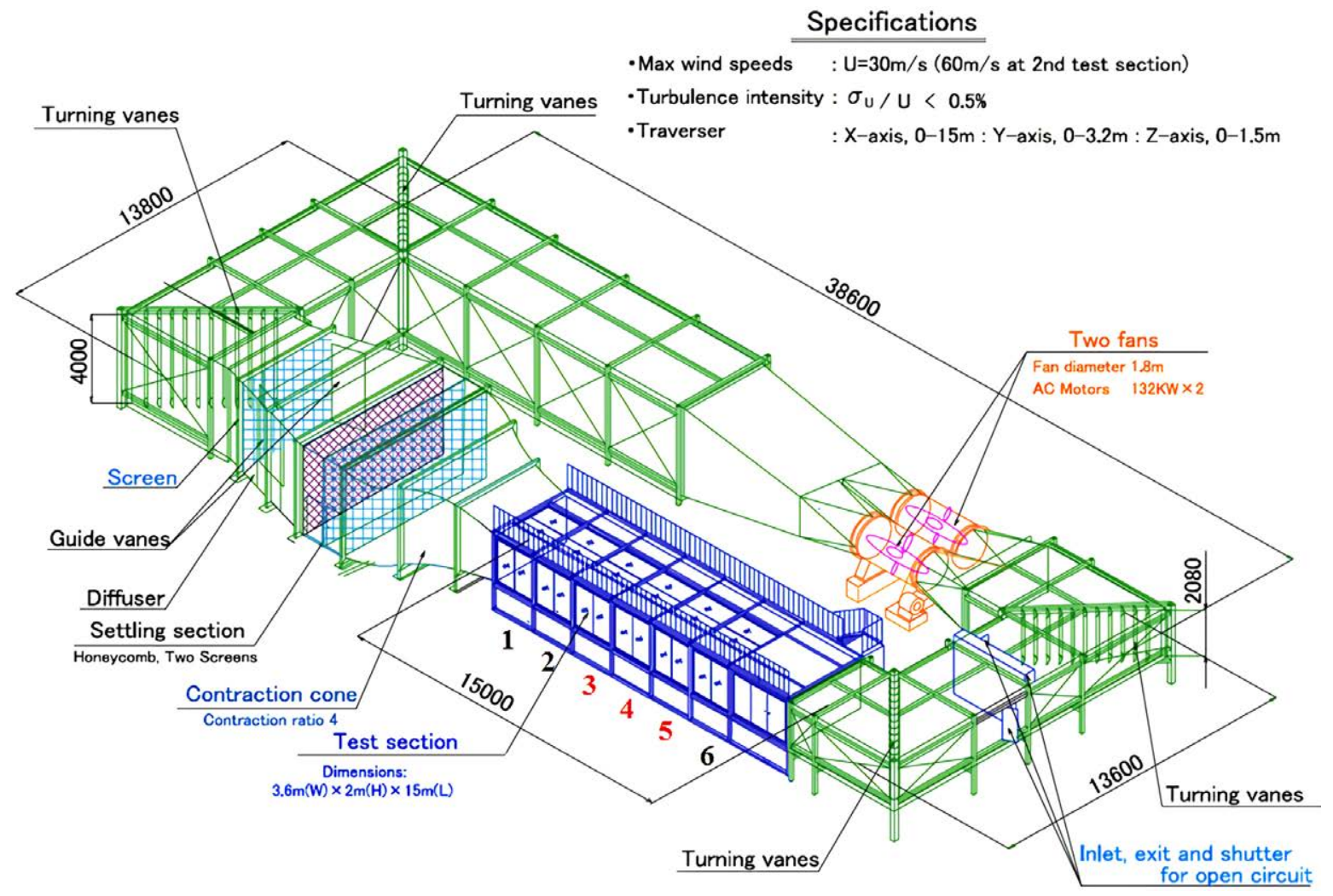

Figura 1. Túnel de viento de la Universidad de Kyushu.

Extraído de Ohya, Miyazaki, Göltenbott, \& Watanabe (2017). 
de $2 \mathrm{~m} / \mathrm{s}$, se anota el voltaje correspondiente que entrega el anemómetro de hilo caliente y esto se repite para valores pares de velocidad del viento hasta 14 $\mathrm{m} / \mathrm{s}$. Luego con los siete pares ordenados se obtiene la recta de mejor ajuste, cuyo coeficiente de determinación, $\mathrm{R}^{2}$, siempre fue mayor que 0.99955 .

La velocidad del viento fue medida con un anemómetro de hilo caliente del fabricante Kanomax Japan Inc. como el que se muestra en la Figura 4. En el experimento realizado se utilizó el modelo 0251R-T5 con un filamento de tungsteno de 5 micrómetros de diámetro. El catálogo se puede consultar en Kanomax (s. f.). El tiempo de medición en cada punto fue de $60 \mathrm{~s}$, la frecuencia de muestreo empleada fue de $1 \mathrm{kHz}$ con un filtro pasa bajos de $200 \mathrm{~Hz}$. Estos valores son los recomendados por el personal técnico y de investigación del RIAM, con base en su experiencia utilizando estos equipos en experimentos similares al que se presenta en este artículo, como por ejemplo en Ohya, \& Karasudani (2010).

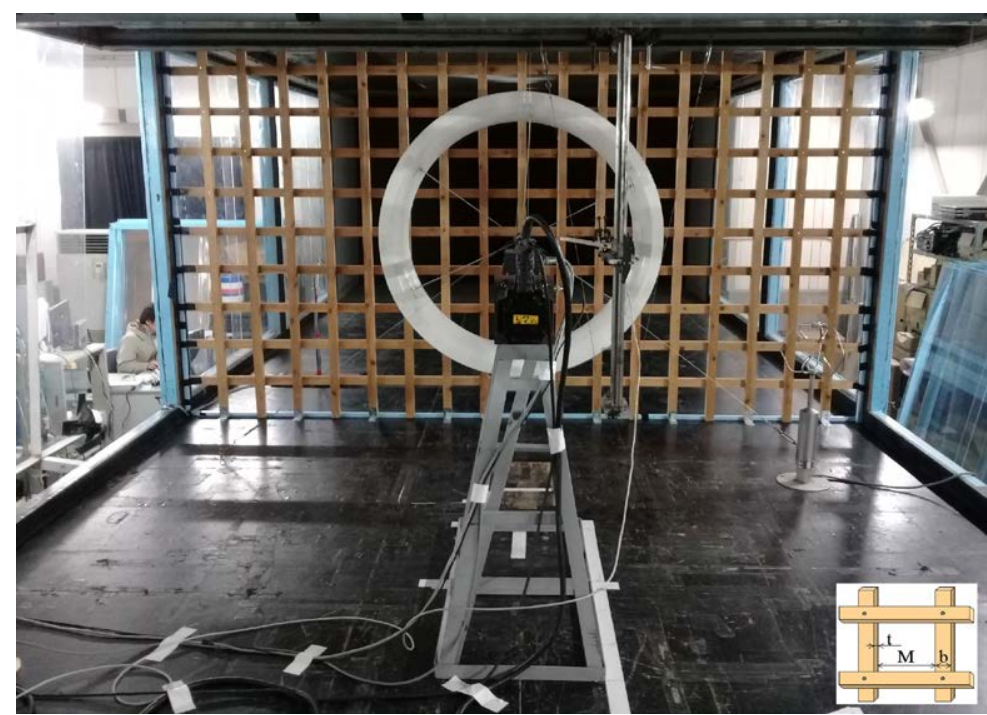

Figura 2. Rejilla para generación de turbulencia. Elaboración propia de la investigación.

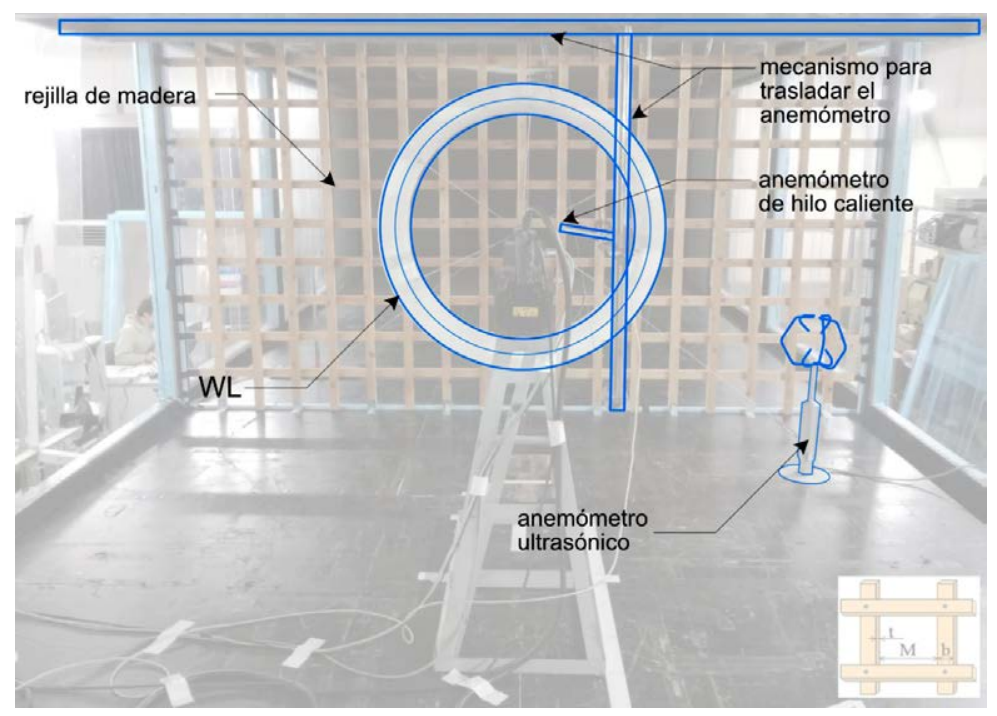

Figura 3. Elementos del montaje experimental. Elaboración propia de la investigación.
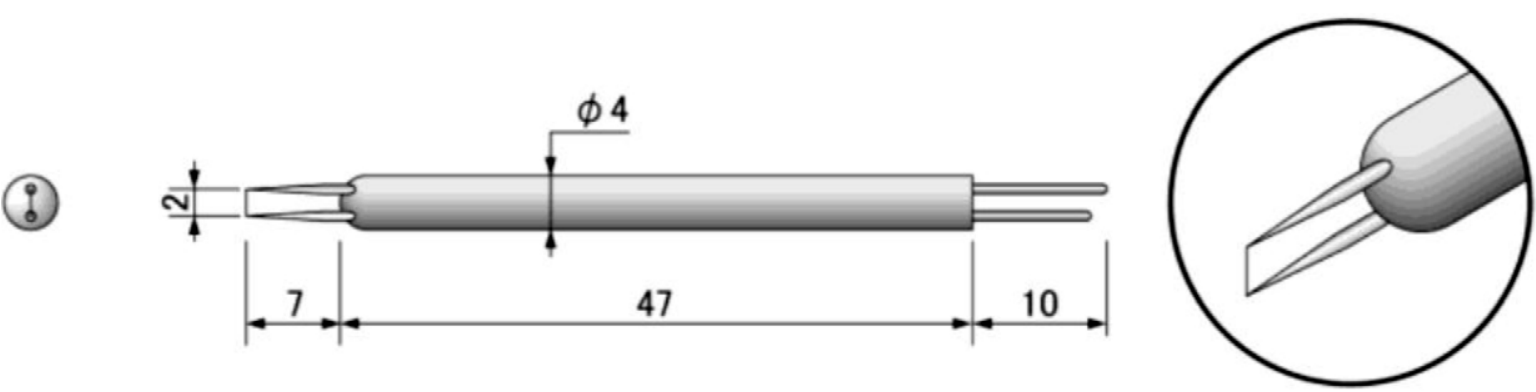

Figura 4. Anemómetro de hilo caliente, acotación en mm.

Extraído de Kanomax (s. f.). 
El WL empleado es el CiiB5, cuya geometría es similar a CiiB10 detallado en Ohya, \& Karasudani (2010), la única diferencia es el valor de h (ver Figura 5), que en el CiiB5 es de $5 \mathrm{~cm}$ y en el CiiB10 es de $10 \mathrm{~cm}$.

viento, sin la rejilla de turbulencia instalada. En segundo lugar, se coloca la rejilla de turbulencia y se mide la intensidad de turbulencia en el eje del túnel de viento. En tercer lugar, se determina el efecto del WL en la velocidad del viento incidente, el cual es flujo turbulento por estar colocada la rejilla.

\section{Velocidad del viento en el eje del túnel sin la rejilla instalada}

Se mide la velocidad del viento en el eje de la sección de pruebas del túnel de viento, con el anemómetro de hilo caliente, sin rejilla de generación de turbulencia y sin ningún WL instalado.

Un esquema de la sección de pruebas y las estaciones de medición se muestra en la Figura 6, cada estación está separada medio metro de la anterior, centrada en la

Los parámetros geométricos del WL CiiB5 empleado en esta investigación e indicados en la Figura 5, se detallan en la Tabla 1.

\section{Pruebas realizadas}

Se realizan tres pruebas, primero se mide la velocidad en el eje del túnel de coordenada vertical y a lo ancho del túnel. En la Figura 6 se incluye la rejilla en la ubicación prevista con fines ilustrativos (en el marco estructural del túnel entre los paneles 2 y 3 ), pero en esta prueba la rejilla no estaba colocada durante las mediciones.

Tabla 1. Parámetros geométricos del Wind Lens CiiB5

\begin{tabular}{lcc}
\hline \multicolumn{1}{c}{ Parámetro geométrico } & Identificador en la Figura 5 & Cota (mm) \\
\hline Diámetro exterior parte trasera (incluyendo el anillo) & $\mathrm{D}_{\text {brim }}$ & 1263 \\
Diámetro exterior parte frontal & $\mathrm{D}_{\text {in }}$ & 1080 \\
Separación de las aspas respecto a la estructura (se & $\mathrm{S}$ & 13 \\
obtienen valores entre 5 y $20 \mathrm{~mm}$, se da un promedio) & & \\
Ancho del anillo posterior & $\mathrm{h}$ & 50 \\
Longitud del concentrador & $\mathrm{Lt}$ & 224 \\
Ubicación del aspa & $\mathrm{L}$ & 107 \\
Diámetro interior libre & $\mathrm{D}$ & 1030 \\
\hline
\end{tabular}

Nota: Fuente propia de la investigación. 


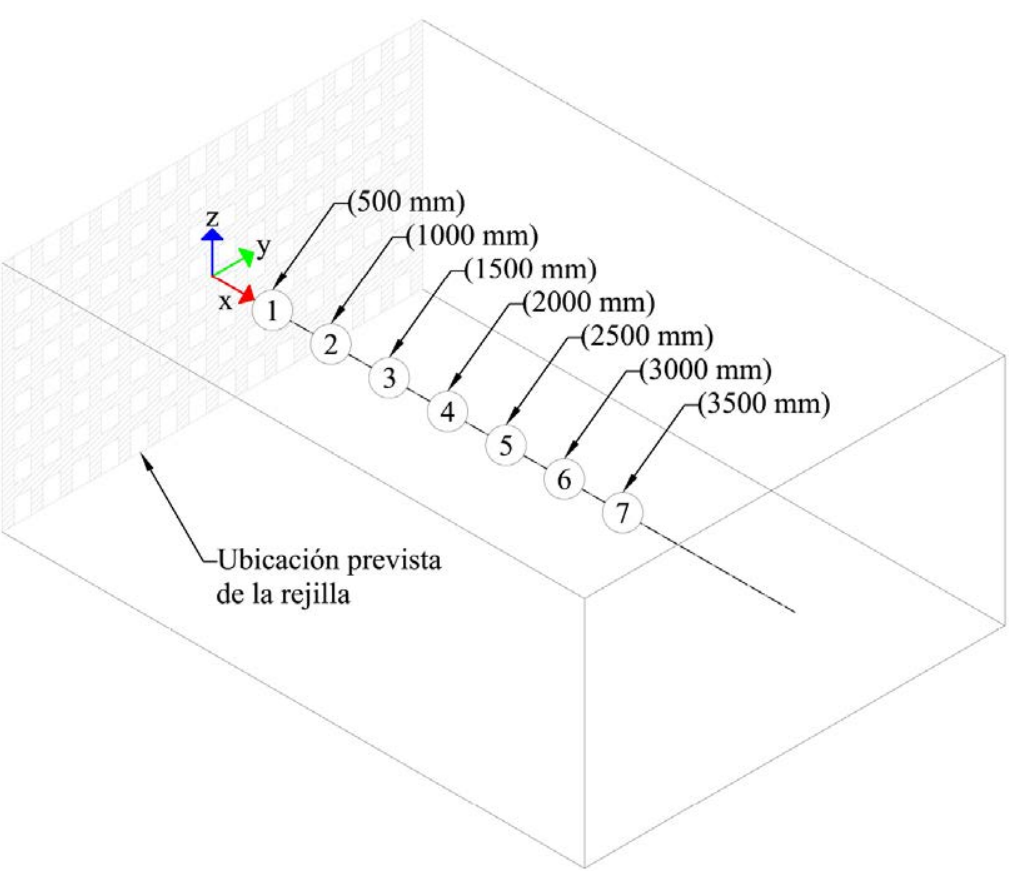

Figura 6. Estaciones de medición de la velocidad en el eje del túnel.

Elaboración propia de la investigación.

\section{Efecto del wind lens en la velocidad del viento en flujo turbulento}

El efecto del WL en la velocidad del flujo se mide en cuatro estaciones donde la IT tiene valores de interés, estos son $5 \%, 10 \%, 15 \%$ y el valor máximo dentro de los límites del experimento, que corresponde a $28.6 \%$.

Considerando los resultados de las mediciones descritas anteriormente, las coordenadas de estas estaciones, desde la rejilla de turbulencia, son respectivamente $3.1 \mathrm{~m}$, $1.32 \mathrm{~m}, 0.93 \mathrm{~m}$ y $0.5 \mathrm{~m}$. En la Figura 7 se muestra de forma esquemática la ubicación de las cuatro estaciones donde se

\section{Intensidad de tur- bulencia en el eje del túnel de viento con la rejilla instalada}

Se coloca la rejilla para generar turbulencia en el marco estructural del túnel entre los paneles 2 y 3 y se mide el valor de la velocidad del viento con el anemómetro de hilo caliente, para calcular la intensidad de turbulencia se utiliza la ecuación 1.

Las mediciones se realizan en las mismas siete estaciones a lo largo del eje del túnel, mostradas en la Figura 6 , las cuales coinciden con las siete estaciones donde se midió la velocidad del viento en el túnel sin la rejilla de turbulencia.

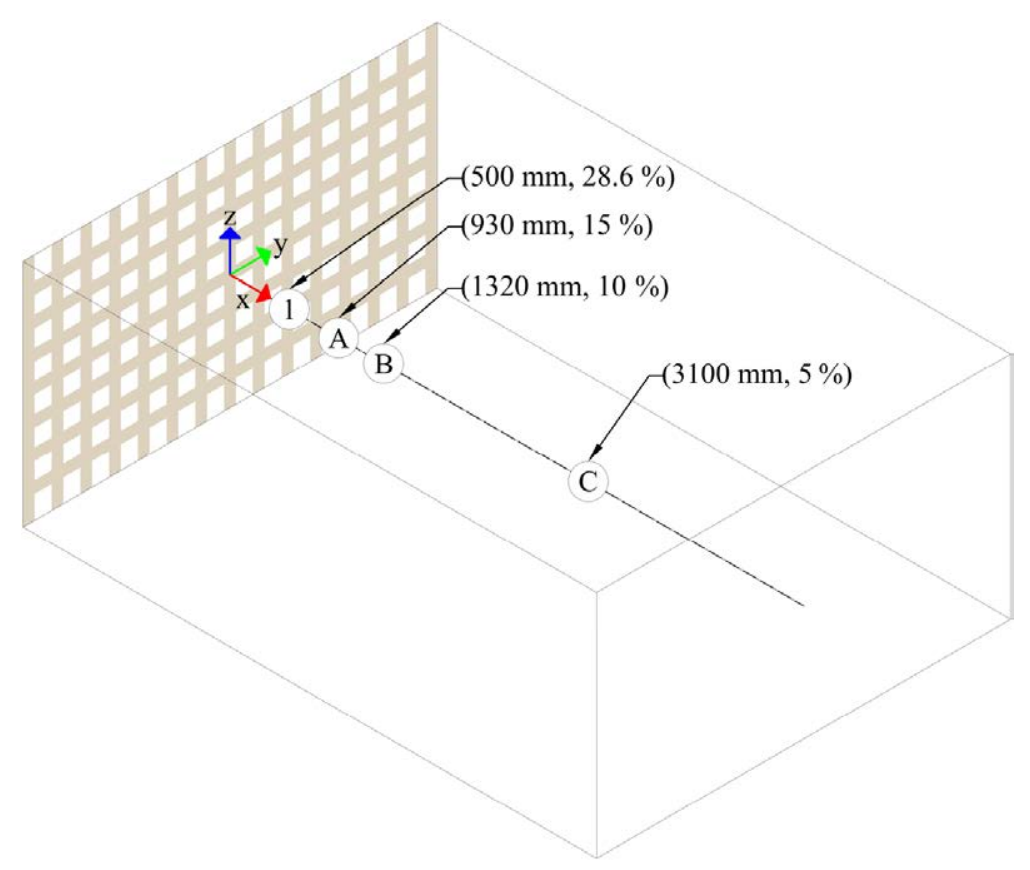

Figura 7. Estaciones donde se medirá el efecto del WL en el flujo turbulento.

Elaboración propia de la investigación. 
mide el efecto del WL en el flujo turbulento, con los valores correspondientes de distancia desde la rejilla de turbulencia y el valor de IT.

La nomenclatura de la Figura 7 mantiene el número 1 en la estación más cercana a la rejilla porque coincide con la estación 1 de las siete donde se realizaron las mediciones iniciales. Se nombran como A, B y C (en las coordenadas $\mathrm{x}=930 \mathrm{~mm}, \mathrm{x}=1320 \mathrm{~mm}$ $\mathrm{y} x=3100 \mathrm{~mm}$, respectivamente) las otras tres estaciones donde se medirá el efecto del WL en el flujo turbulento. En el caso de A, $\mathrm{B}$ y $\mathrm{C}$, sus coordenadas no coinciden con ninguna de las siete estaciones donde se realizaron las mediciones iniciales.
Para determinar propiamente el efecto del WL sobre el flujo, se mide la velocidad del viento con el anemómetro de hilo caliente en cinco puntos, para cada una de las estaciones 1, A, B y C; tanto con el WL como sin el WL. En cada estación se abarca una distancia de $500 \mathrm{~mm}$ aguas arriba del plano del rotor que operaría dentro del WL. La distancia radial se define a $75 \%$ del radio del rotor, que para el WL CiiB5 sería de 500 $\mathrm{mm}$, por lo que el anemómetro se coloca a $375 \mathrm{~mm}$ del eje del WL. Esta configuración se muestra en la figura 8, para el caso de la estación 1. El posicionamiento del sensor a $75 \%$ del radio del rotor obedece a las

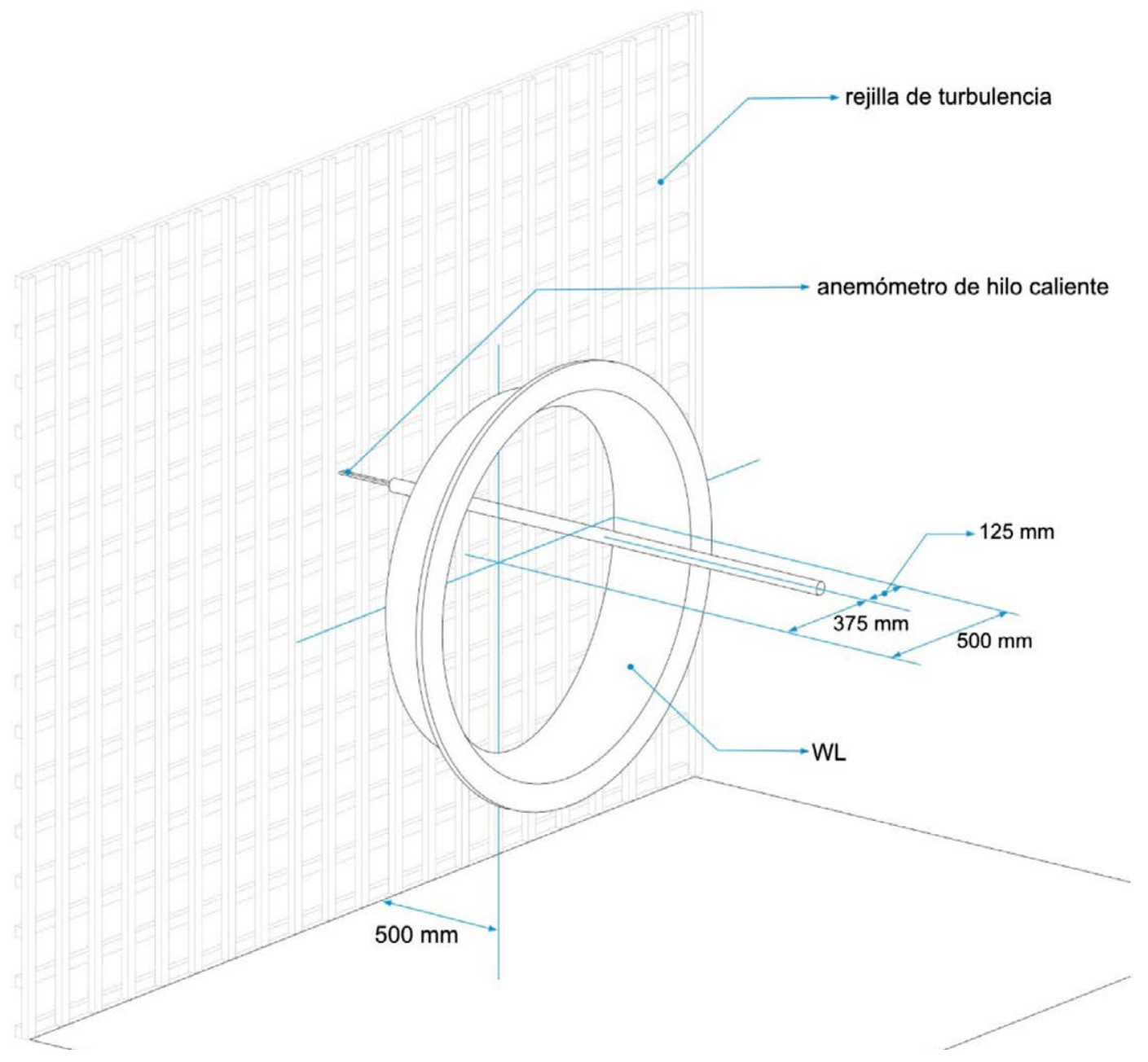

Figura 8. Perspectiva axonométrica de la colocación el hilo caliente dentro del WL. Elaboración propia de la investigación. 
restricciones impuestas por los soportes del mecanismo para trasladar el anemómetro de hilo caliente (Figuras 2 y 3), de modo que solo en esa posición es posible que el anemómetro sobresalga del WL aguas arriba, hasta las coordenadas de interés.

Dado que en la estación 1 el WL está precisamente a $500 \mathrm{~mm}$ de la rejilla de turbulencia, solo se mide el viento aguas arriba hasta $450 \mathrm{~mm}$ de distancia del WL, como se muestra en la Figura 9. En las estaciones A, B y C la separación entre cada uno de los cinco puntos de medición fue de $125 \mathrm{~mm}$. Tanto en la Figura 8 como en la Figura 9 se muestra el WL, pero las mediciones se realizaron con el WL y sin este, precisamente para determinar el efecto de la presencia del WL en el flujo turbulento.

\section{Análisis y resultados}

Los resultados y su análisis se dividen en las mismas tres secciones que se plantearon para la ejecución de las pruebas, a saber, velocidad del viento en el eje del túnel sin la rejilla instalada, intensidad de turbulencia en el eje del túnel de viento con la rejilla instalada y efecto del WL en la velocidad del viento en flujo turbulento.

En el primer caso se presenta una gráfica de la velocidad del viento, que es sumamente estable a lo largo del túnel de viento, con una magnitud alrededor de $9.6 \mathrm{~m} / \mathrm{s}$.

En el segundo caso se muestra la gráfica de IT y cómo esta decae de forma asintótica conforme se realizan mediciones alejándose de la rejilla de turbulencia.

\section{Vista lateral}

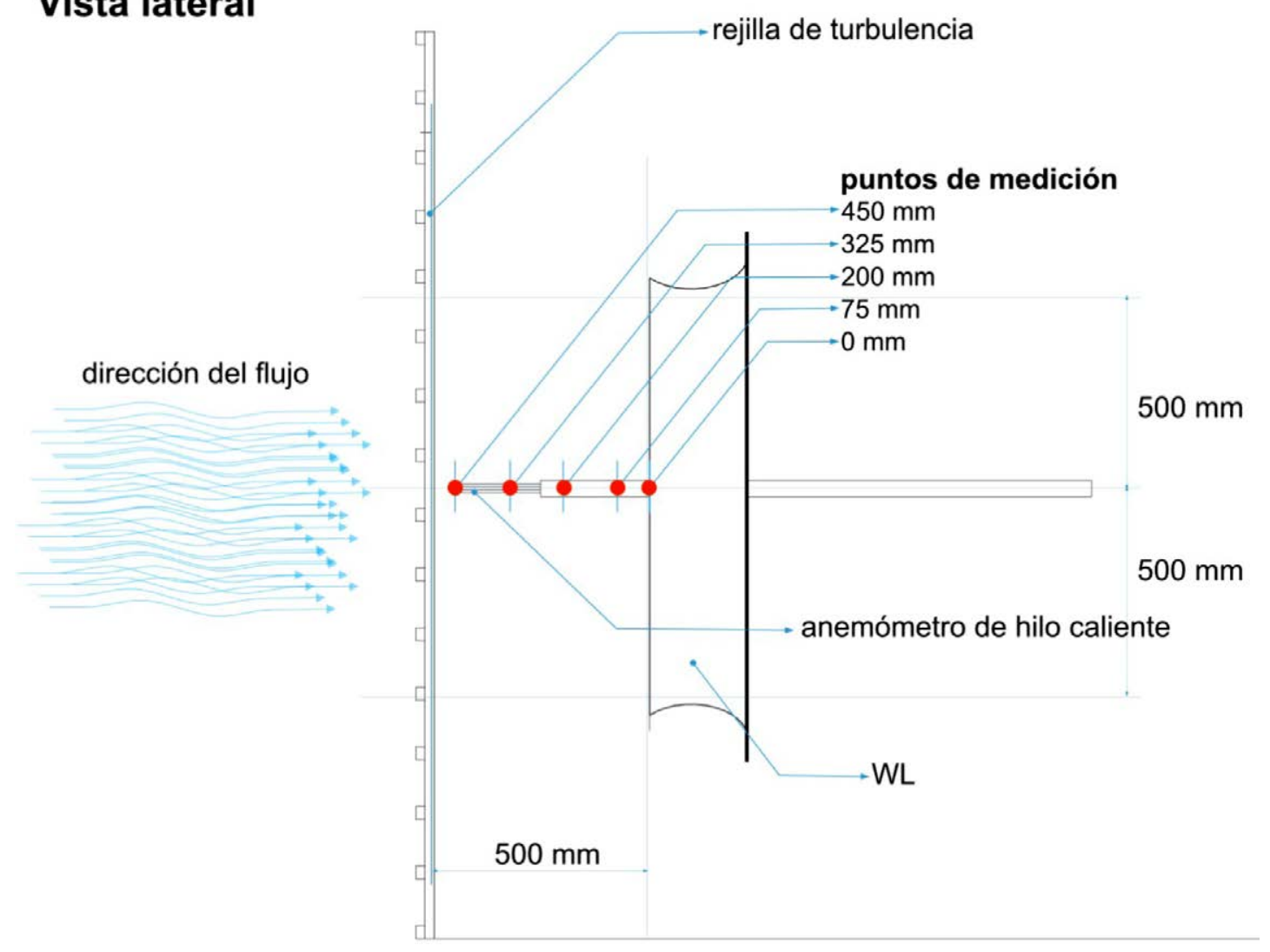

Figura 9. Puntos donde se medirá el efecto del WL en el flujo turbulento, estación 1. Elaboración propia de la investigación. 
En el tercer caso se detalla cómo el WL genera un aumento en la velocidad del viento en flujo turbulento. Se presenta una gráfica compuesta por las mediciones en las cuatro estaciones de interés, en la que se observa que el efecto del WL es mayor en las estaciones de mayor turbulencia.

A continuación, se detallan los resultados de cada prueba realizada.

\section{Velocidad del viento en el eje del túnel sin la rejilla instalada}

Los resultados de la velocidad del viento medida en el eje del túnel sin rejilla de turbulencia, en las siete estaciones indicadas en la Figura 6, se muestran en la Gráfica 1. En la cual se ha colocado la rejilla con fines ilustrativos, pues su posición prevista define el origen del sistema de coordenadas, el cual está 4 metros aguas abajo del inicio de la zona de pruebas del túnel de viento. Debe notarse que en la Gráfica 1 el eje vertical es la velocidad del viento en metros por segundo y no la coordenada vertical del túnel de viento, esta construcción artificial de superponer la rejilla de turbulencia se ha hecho para poder comparar los resultados de esta gráfica con los obtenidos en las secciones posteriores, manteniendo un mismo formato en la presentación de los resultados.

La estación de menor velocidad en la Gráfica 1 es de $9.57 \mathrm{~m} / \mathrm{s}$, en la posición de 2 $000 \mathrm{~mm}$, y coincide con la transición entre el panel lateral 3 (parcialmente removido) y el panel lateral 4 (totalmente removido). Esto explica por qué esa es la estación que tiene una ligera disminución en la velocidad respecto de sus vecinas.

En las otras tres mediciones aguas abajo, se observa una ligera recuperación de la velocidad, esto porque el túnel es de circuito cerrado y en esas posiciones debe estar regresando el aire que salió por los paneles removidos.

La mayor velocidad registrada en el túnel sin rejilla es de $9.76 \mathrm{~m} / \mathrm{s}$ y corresponde con la coordenada de $500 \mathrm{~mm}$, lo cual es de esperarse, pues esa estación es la más cercana a la descarga sin paneles removidos de la sección de pruebas, de modo que por ahí debe pasar la mayor cantidad de viento en comparación al resto.

Gráfica 1. Velocidad del viento en el eje del túnel sin rejilla de turbulencia

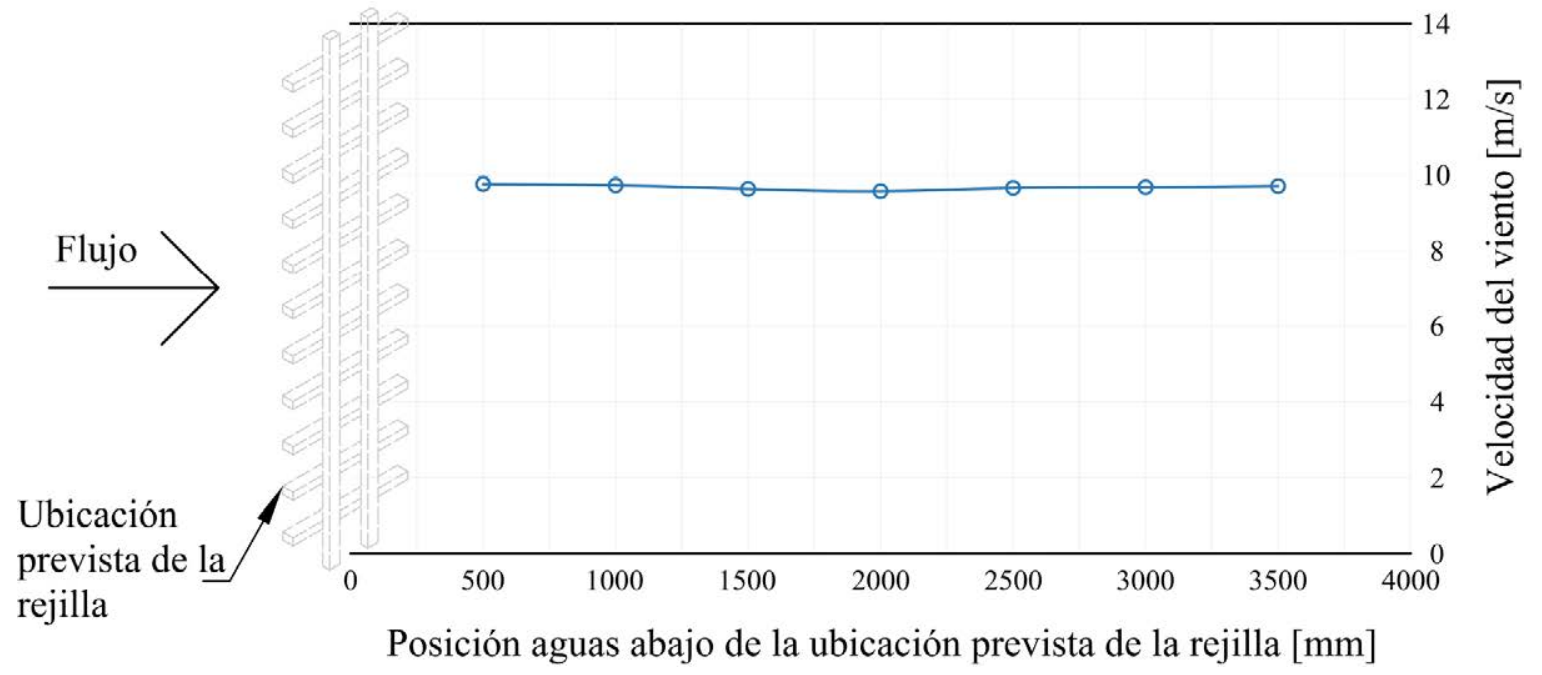

Nota: Fuente propia de la investigación. 
La velocidad mínima obtenida es ligeramente mayor que $98 \%$ de la velocidad máxima, por lo que se puede afirmar que hay una variación de menos del $2 \%$ en la velocidad en el túnel de viento en el experimento, en las condiciones descritas en este artículo.

\section{Intensidad de turbulencia en el eje del túnel de viento con la rejilla instalada}

Los resultados de IT, calculada según la ecuación 1 , con base en las mediciones de velocidad de viento realizadas en las siete estaciones indicadas en la Figura 6, se muestran en la Gráfica 2. En este caso las mediciones se tomaron con la rejilla de turbulencia instalada. Debe notarse que al igual que en la Gráfica 1, el eje vertical no es la coordenada vertical del túnel, en este caso es la intensidad de turbulencia.

De los resultados mostrados en la Gráfica 2, se obtienen las cuatro estaciones donde se medirá el efecto del WL en el flujo turbulento (ver Figura 7), que corresponden a valores de IT de $5 \%, 10 \%, 15 \%$ y $28.6 \%$.
La turbulencia se genera al pasar el viento por la rejilla, donde se pierde el estado de flujo turbulento desarrollado de baja IT, menor que $0.5 \%$ de acuerdo con Ohya, Miyazaki, Göltenbott, \& Watanabe (2017). Esta condición de flujo uniforme y suave se impone por las mallas con forma de panal de abejas que son parte del túnel y están diseñadas para ese fin, indicadas en la Figura 1 en el "Settling section".

Considerando que la turbulencia es un fenómeno que requiere energía para generarse y que en la naturaleza los procesos y sistemas siempre tienden al estado de menor energía posible, es de esperar que la IT decaiga al alejarse de la rejilla por los procesos de disipación viscosa, que causan que el viento vaya perdiendo el estado de desorden y tienda a ser menor la desviación estándar de las mediciones, conforme se avanza agua abajo.

La rejilla genera una alta turbulencia en sus alrededores, pero esta es rápidamente amortiguada, debido a que la fricción interna es proporcional a la IT, este rápido decaimiento es evidente en la Gráfica 2.

Gráfica 2. Intensidad de turbulencia en el eje del túnel con rejilla de turbulencia

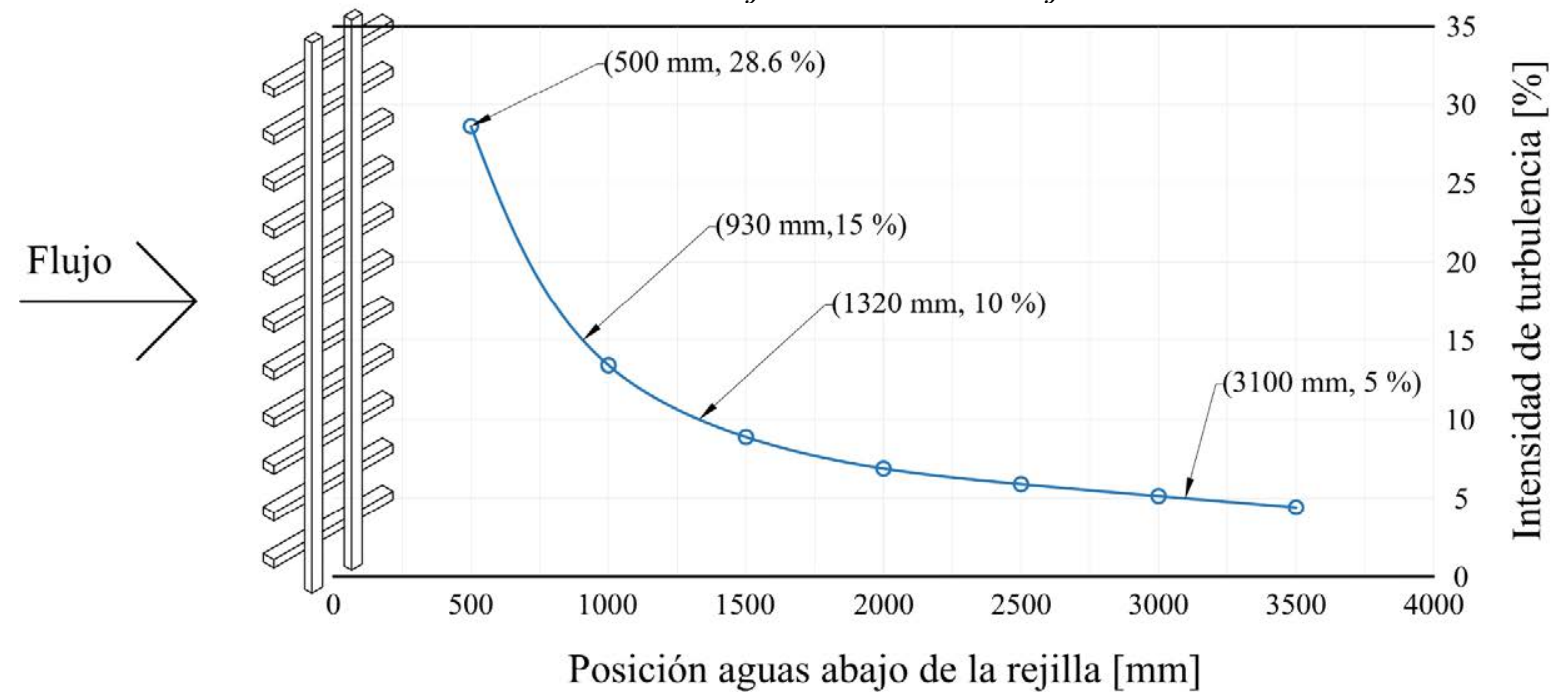

Nota: Fuente propia de la investigación. 
A una distancia de $1500 \mathrm{~mm}$ de la rejilla, la pendiente es más suave, aunque siempre decreciente, pues al ser menor la IT el proceso de disipación viscosa tiene menor efecto sobre el flujo, por lo que se dará un comportamiento asintótico a un flujo turbulento desarrollado de baja IT.

\section{Efecto del wind lens en la velocidad del viento en flujo turbulento}

Una vez realizadas las pruebas descritas anteriormente, es posible determinar el efecto del WL sobre el flujo turbulento, en las estaciones donde la IT tiene valores de $5 \%, 10 \%, 15 \%$ y $28.6 \%$, mostradas en la Figura 7 y Gráfica 2.

La Gráfica 3 muestra, de forma combinada, el efecto del WL en el flujo turbulento, al realizar las mediciones en las cuatro estaciones de interés. La escala vertical es la velocidad del viento y coincide con la mostrada en la Gráfica 1. La escala horizontal se ha superpuesto artificialmente a conveniencia. Se han colocado los resultados de las cuatro pruebas en la misma gráfica para comparar de mejor forma el efecto del WL en el flujo, sin embargo, cada prueba fue realizada de forma independiente y excluyente, es decir, cuando el WL estaba en $\mathrm{x}=500 \mathrm{~mm}$, no había ningún otro WL en el túnel; lo mismo aplica para las otras tres estaciones. También se debe destacar que, aunque en la Gráfica 3 aparece la silueta del WL, las mediciones fueron realizadas en los mismos puntos con el WL y sin este, precisamente para determinar el efecto del concentrador de flujo en la velocidad del viento.

En las cuatro estaciones analizadas resulta evidente que el WL aumenta la velocidad del viento, también es notorio que el aumento ocurre de forma progresiva conforme el flujo se aproxima al WL. En los casos de IT $=5 \%$ y $10 \%$, el WL, genera una velocidad en el plano del rotor de 12 $\mathrm{m} / \mathrm{s}$, siendo $20 \%$ mayor que la velocidad en el mismo plano en ausencia del WL, que ronda $\operatorname{los} 10 \mathrm{~m} / \mathrm{s}$.

En las estaciones de mayor IT el efecto del WL es mayor, lo cual se debe analizar de dos maneras. Por una parte, el flujo aún sin WL se va recuperando luego de pasar por la rejilla, el caudal luego del WL debe ser el mismo que antes del WL, pero el desorden en el flujo causa que existan regiones con mayor o menor velocidad, asociado

Gráfica 3. Efecto del wind lens en cuatro estaciones en flujo turbulento

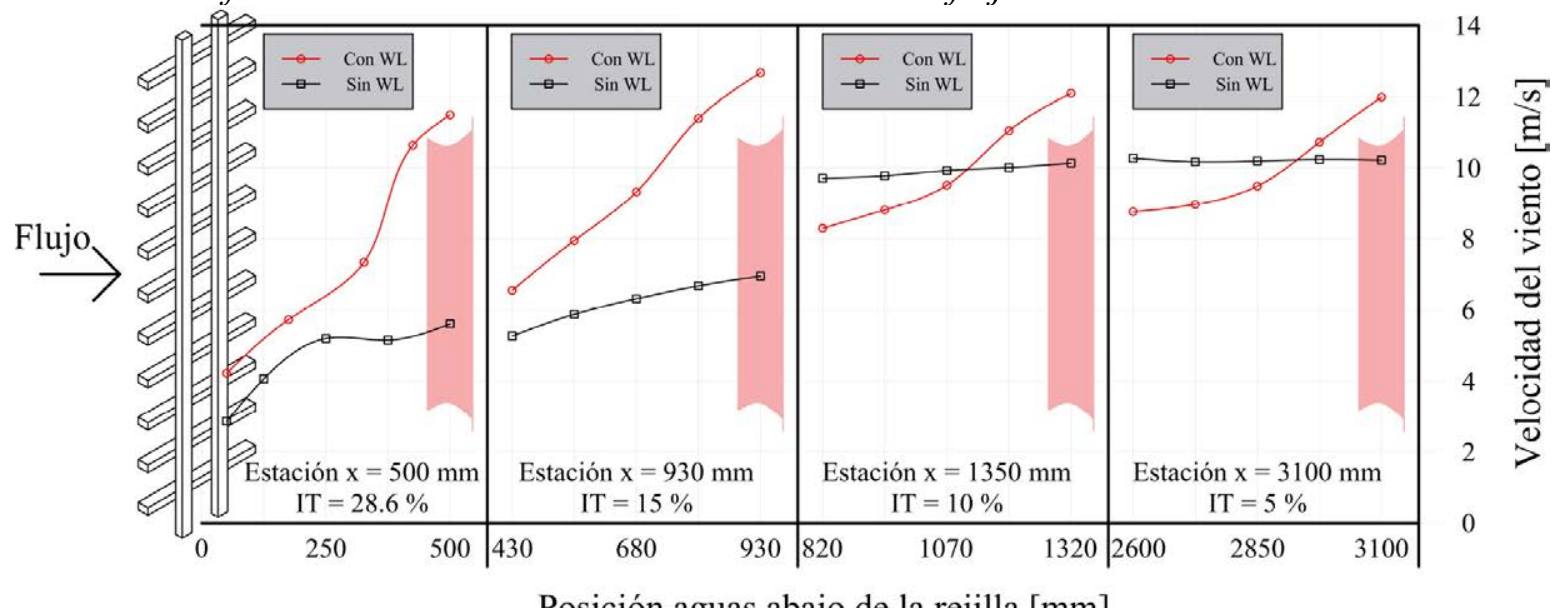

Posición aguas abajo de la rejilla $[\mathrm{mm}]$

Nota: Fuente propia de la investigación. 
precisamente a un aumento en la turbulencia. En las estaciones más alejadas de la rejilla el flujo está más ordenado y se tiende al comportamiento mostrado en la Gráfica 1. De este modo, parte del aumento de la velocidad del flujo en las estaciones con mayor IT no es debido al WL.

Por otra parte, el WL genera una reducción de la presión en su parte posterior, esta reducción de presión se debe al desprendimiento de vórtices que causa el anillo posterior del WL. El desprendimiento de vórtices se ve favorecido en el flujo turbulento, por lo que se puede afirmar, y se confirma con los resultados, que el WL genera un mayor aumento de velocidad en el caso de flujo con mayor IT.

Por la naturaleza del flujo turbulento, los resultados poseen cierta aleatoriedad que se muestra en una falta de continuidad en la recuperación del flujo, particularmente entre las estaciones de $\mathrm{x}=930 \mathrm{~mm} \mathrm{y} \mathrm{x}=1350 \mathrm{~mm}$. Esto puede estar relacionado con el hecho de tomar mediciones únicamente en el eje del túnel y no en un área. Si se tomaran mediciones, sin el WL, y se realizaran promedios en toda el área de sección transversal del túnel, se obtendrían resultados idénticos a los de la Gráfica 1, pues el caudal total de aire no puede disminuir y luego aumentar dentro del túnel, sino que debe ser una constante, a menos que se presentaran efectos de flujo compresible, que están fuera del rango de velocidades de este experimento.

No obstante, con las mediciones realizadas, se puede analizar el efecto del WL en el flujo turbulento, que resulta en un mayor aumento de la velocidad a mayor turbulencia. Al afirmar esto se debe tener en cuenta que las mediciones se hacen siempre sin la turbina eólica presente. Al existir un rotor en movimiento se vería alterado todo el campo de flujo, pues la velocidad del viento disminuye al acercase al rotor, según se explica en la teoría del disco actuador, de modo que este efecto estaría superpuesto al aumento de velocidad, debido al WL.

Otro efecto que está por fuera de este estudio es la variación de la IT, por causa de la presencia del WL, pues los valores de referencia de IT en la Gráfica 3 se midieron sin el difusor en el túnel. Este tipo de análisis corresponderán a un trabajo futuro.

\section{Conclusiones}

Se realizaron mediciones de velocidad del viento en un túnel de viento con dimensiones para albergar turbinas eólicas de hasta $1 \mathrm{~m}$ de diámetro. Se caracterizó la turbulencia dentro la zona de pruebas del túnel al colocar una rejilla de madera, lo que permitió obtener las coordenadas, desde la rejilla, en la cual se registran valores específicos de intensidad de turbulencia.

Se logró determinar cuantitativamente el efecto de un difusor tipo wind lens en un flujo turbulento. Mediante la comparación de la magnitud de la velocidad del viento al aproximarse al plano del rotor de una turbina eólica, en condiciones de intensidad de turbulencia de $5 \%, 10 \%, 15 \%$; y $28.6 \%$, se obtuvo, en todos los casos, un aumento en la velocidad del viento.

Se determinó que el efecto positivo del wind lens en el flujo se ve potenciado por la turbulencia, pues, en las estaciones de mayor intensidad de turbulencia, el aumento en la velocidad del viento fue mayor. Por lo que se concluye que el wind lens es un concentrador de flujo adecuado para turbinas que operan en condiciones de flujo turbulento.

En las estaciones de menor intensidad de turbulencia, de $5 \%$ y $10 \%$, el flujo es más estable y el wind lens produce un aumento en la velocidad del $20 \%$. 
Sin embargo, no se puede afirmar que ese $20 \%$ de aumento ocurrirá en condiciones reales de operación, donde la presencia de un rotor en movimiento interactúa con el campo de flujo.

\section{Reconocimientos}

Al estudiante Emanuel Fallas Hernández por su aporte a este documento, al Instituto Tecnológico de Costa Rica por el apoyo a esta investigación y al Instituto de Investigación en Mecánica Aplicada (RIAM por sus siglas en inglés Research Institute for Applied Mechanics) de la Universidad de Kyushu, Japón, por facilitar las instalaciones para la realización de la parte experimental de esta investigación.

\section{Conflicto de intereses}

Los autores declaran no tener conflicto de interés.

\section{Declaración de la contribu- ción de los autores}

Todos los autores afirmamos que se leyó y aprobó la versión final de este artículo.

El porcentaje total de contribución para la conceptualización, preparación y corrección de este artículo fue el siguiente: G.R.N. 70 \%, P.C.T. 15 \% y F.H.C. $15 \%$.

\section{Declaración de disponibilidad de los datos}

Los datos que respaldan los resultados de este estudio serán puestos a disposición por el autor G.R.N., previa solicitud razonable.

\section{Referencias}

Aouini, A. (2012). System for Converting Wind Energy. WO2012039688 -WIPO IP PORTAL. [Patent]. https://bit.ly/3303haj

Burton, T., Jenkins, N., Sharpe, D., \& Bossanyi, E. (2011). Wind Energy Handbook. https://dx. doi.org/10.1002/9781119992714

Clements, L., \& Chowdhury, A. (2019). Performance evaluation of wind lens in turbulent environment. Energy Procedia, 160, 777-782. https:// dx.doi.org/10.1016/j.egypro.2019.02.161

El-Shahat, A., Hasan, M.-M., \& Wu, Y., (2018). Vortex Bladeless Wind Generator for Nano-Grids. 2018 IEEE Global Humanitarian Technology Conference (GHTC). https://dx. doi.org/10.1109/GHTC.2018.8601572

Göltenbott, U., Ohya, Y., Yoshida, S., \& Jamieson, P. (2017). Aerodynamic interaction of diffuser augmented wind turbines in multi-rotor systems. Renewable Energy, 112, 25-34. https:// dx.doi.org/10.1016/j.renene.2017.05.014

Hashem, I., Mohamed, M. H., \& Hafiz, A. A. (2017). Aero-acoustics noise assessment for WindLens turbine. Energy, 118, 345-368. https:// dx.doi.org/10.1016/j.energy.2016.12.049

Heikal, H.A. et al., (2018). On the actual power coefficient by theoretical developing of the diffuser flange of wind-lens turbine. Renewable Energy, 125, 295-305. https://dx.doi.or$\mathrm{g} / 10.1016 / \mathrm{j}$.renene.2018.02.100

Hu, J.-F., \& Wang, W.-X. (2015). Upgrading a Shrouded Wind Turbine with a Self-Adaptive Flanged Diffuser. Energies, 8(6), 5319-5337. https://dx.doi.org/10.3390/en8065319

Jang, H., Kim, D., Hwang, Y., Paek, I., Kim, S., \& Baek, J. (2019). Analysis of Archimedes Spiral Wind Turbine Performance by Simulation and Field Test. Energies, 12(24), 4624. https://dx.doi.org/10.3390/en12244624

Kanomax. (s. f.). Hot-Wire Anemometer Smart CTA. Model 7250. https:/www.kanomax.co.jp/ img_data/file_731_1550041294.pdf

Keramat Siavash, N., Najafi, G., Tavakkoli Hashjin, T., Ghobadian, B., \& Mahmoodi, E. (2020). Mathematical modeling of a horizontal axis shrouded wind turbine. Renewable Energy, 146, 856-866. https://dx.doi.org/10.1016/j. renene.2019.07.022

Khamlaj, T. A., \& Rumpfkeil, M. P. (2018). Analysis and optimization of ducted wind turbines. 
Energy, 162, 1234-1252. https://dx.doi.org/10.1016/j.energy.2018.08.106

Kosasih, B., \& Saleh Hudin, H. (2016). Influence of inflow turbulence intensity on the performance of bare and diffuser-augmented micro wind turbine model. Renewable Energy, 87, 154-167. https://dx.doi.org/10.1016/j.renene.2015.10.013

Maftouni, N., \& Parsa, H. (2019). Effects of Implementing a Diffuser around the Wind Turbine. 2019 International Conference on Power Generation Systems and Renewable Energy Technologies (PGSRET). https://dx.doi. org/10.1109/PGSRET.2019.8882727

Nasution, A., \& Purwanto, D. W. (2011). Optimized curvature interior profile for Diffuser Augmented Wind Turbine (DAWT) to increase its energy-conversion performance. 2011 IEEE Conference on Clean Energy and Technology (CET). http://dx.doi.org/10.1109/ CET.2011.6041483

Ohya, Y.(2019). Multi-Rotor Systems Using Five Ducted Wind Turbines for Power Output Increase (Multi Lens Turbine). AIAA Scitech Forum. http://dx.doi.org/10.2514/6.2019-1296

Ohya, Y., \& Karasudani, T. (2010). A Shrouded Wind Turbine Generating High Output Power with Wind-lens Technology. Energies, 3(4), 634 649. https://doi.org/10.3390/en3040634

Ohya, Y., Karasudani, T., Nagai, T., \& Watanabe, K. (2017). Wind lens technology and its application to wind and water turbine and beyond. Renewable Energy and Environmental Sustainability, 2, 2. http://dx.doi.org/10.1051/ rees/2016022

Ohya, Y., Miyazaki, J., Göltenbott, U., \& Watanabe, K. (2017). Power Augmentation of Shrouded Wind Turbines in a Multirotor System. Journal of Energy Resources Technology, 139(5). http://dx.doi.org/10.1115/1.4035754

Richmond-Navarro, G., Calderon-Munoz, W. R., LeBoeuf, R., \& Castillo, P. (2017). A Magnus Wind Turbine Power Model Based on Direct Solutions Using the Blade Element Momentum Theory and Symbolic Regression.
IEEE Transactions on Sustainable Energy, 8(1), 425-430. https://dx.doi.org/10.1109/ TSTE.2016.2604082

Riyanto, Pambudi, N. A., Febriyanto, R., Wibowo, K. M., Setyawan, N. D., Wardani, N. S., ... Rudiyanto, B. (2019). The Performance of Shrouded Wind Turbine at Low Wind Speed Condition. Energy Procedia, 158, 260-265. http:// dx.doi.org/10.1016/j.egypro.2019.01.086

SAGE Journals. (2006). Book Review: Wind Energy in the Built Environment - Concentrator Effects of Buildings. Wind Engineering, 30(5), 451-452. http://dx.doi. org/10.1260/030952406779502623

Takahashi, S., Hata, Y., Ohya, Y., Karasudani, T., \& Uchida, T. (2012). Behavior of the Blade Tip Vortices of a Wind Turbine Equipped with a Brimmed-Diffuser Shroud. Energies, 5(12), 5229-5242. http://dx.doi.org/10.3390/en5125229

Wang, W.-X., Matsubara, T., Hu, J., Odahara, S., Nagai, T., Karasutani, T., \& Ohya, Y. (2015). Experimental investigation into the influence of the flanged diffuser on the dynamic behavior of CFRP blade of a shrouded wind turbine. Renewable Energy, 78, 386-397. http://dx. doi.org/10.1016/j.renene.2015.01.028

Watanabe, K., Fukutomi, S., Ohya, Y., \& Uchida, T. (2020). An Ignored Wind Generates More Electricity: A Solar Updraft Tower to a Wind Solar Tower. International Journal of Photoenergy, 2020, 1-9. https://dx.doi. org $/ 10.1155 / 2020 / 4065359$

Yuji, O., \& Koichi, W. (2019). A New Approach Toward Power Output Enhancement Using Multirotor Systems With Shrouded Wind Turbines. Journal of Energy Resources Technology, 141(5). http://dx.doi. org $/ 10.1115 / 1.4042235$

Zhu, H., Sueyoshi, M., Hu, C., \& Yoshida, S. (2019). A study on a floating type shrouded wind turbine: Design, modeling and analysis. Renewable Energy, 134, 1099-1113. https://doi. org/10.1016/j.renene.2018.09.028

\section{(c) $(1 \otimes \Theta$}

Efecto de un difusor tipo wind lens en flujo turbulento (Gustavo Richmond-Navarro, Pedro Casanova-Treto, Franklin Hernández-Castro) Uniciencia is protected by Attribution-NonCommercial-NoDerivs 3.0 Unported (CC BY-NC-ND 3.0) 